\title{
Aortic stiffness and polymorphisms of collagen-I type la gene in COPD patients
}

\begin{abstract}
Currently the connection between chronic obstructive respiratory disease (COPD) and some cardiovascular and cerebrovascular diseases has been established. Vascular disorders in COPD become evident in the early stages of disease and can manifest from endothelial dysfunction to aortic mechanical property disturbance and cerebral circulation disorders. COPD is also known as a disease associated with smoking. A number of researchers have described pathophysiological mechanisms of vascular disorders and in particular arterial stiffening in COPD. A variety of theories have been proposed to explain these observations. Pathophysiological processes that increase arterial stiffness (both physiological and pathophysiological) involve remodeling cascades leading to disturbance of the structural and functional relationships between collagen and elastin in the arterial vascular wall. The main processes involved appear to be inflammatory, proteases and oxidative stress. New research suggests that the vascular dysfunction observed in COPD is associated with some specific genes. New data suggests that this is not just due to endothelial dysfunction, but a violation of the mechanical properties of the arterial vasculature, which is now considered an important component of the pathogenesis of COPD. Surplus aortic stiffness can contribute increasingly to cardiovascular event risk in COPD and is associated with changes in normal cerebral circulation and cognitive function. Research is focused on the study of genetic predisposition for vascular dysfunction in COPD. Various polymorphisms determining the connective tissue metabolism, production of nitric oxide, detoxification of xenobiotics and many others are now all thought relevant. Increasing of aortic stiffness is an important component of the amplification of cardiovascular risk events, coronary disorders and cerebral circulation in patients with COPD. Some polymorphisms are considered especially relevant, such as COL1A1 polymorphisms. It is now important to take into consideration the pathophysiological consequences of increasing arterial stiffness in COPD, which are schematically represented in our review.
\end{abstract}

Keywords: endothelial dysfunction, arterial stiffness, aorta, chronic obstructive pulmonary disease, genotype, cardiovascular risk, pathophysiology
Volume 5 Issue 3 - 2018

Tatiana Brodskaya,Vera Nevzorova, Natalia Zakharchuk, Nina Repina

Therapy department, Pacific State Medical University, Russia

Correspondence: Natalia Zakharchuk, Therapy department, Pacific State Medical University, Ostryakovast, 2, Vladivostok, Russia, Tel 83232451702, Fax 83232451702,

Email zaharchuknat@mail.ru

Received: December 12, 2017 | Published: May 16, 2018
Abbreviations: COPD, chronic obstructive pulmonary disease; COL1A1, collagen-1 type 1a gene; LV, left ventricular

\section{Introduction}

Chronic obstructive pulmonary disease (COPD) pathogenesis is now thought to be a by-product of vascular disturbances. There are data that support that the increasing arterial stiffness observed in COPD plays a role in its pathogenesis. These data can help explain the historical observation of COPD with cardiovascular pathology. Several research publications described pathophysiological mechanisms of arterial stiffening in COPD and understandings of specific mechanisms remain mixed. ${ }^{1-3}$ Association of impaired pulmonary function with cardiovascular morbidity and mortality has also been reported in several studies. ${ }^{4,5}$ However, the nature of this association and the underlying mechanisms are also varied.

The current hypothesis describes aortic stiffness as an independent predictor of total and cardio-vascular mortality and it is thought to be a more important factor than other "classic" risk factors such as blood pressure and total cholesterol. ${ }^{6,7}$ Attenuation of elastic quality of the aorta is attributed to aging, so the degree of aortic stiffness appears to more closely correspond to the biological age of person. ${ }^{8,9}$
It is now well documented that a given gene's polymorphism is one of the principal factors initiating disturbance of mechanical features of the aorta ${ }^{6,10}$ There are many genes associated with amplification of aorta stiffness, as a result many scientists believe that this process is multifactorial. The gene-candidates connected with an abundance of aortic stiffness are genes responsible for angiotensin-converting enzyme, collagen-1a, fibrillin-1, angiotensin-1, endothelin-receptors A and B etc. ${ }^{10-12}$ The passage of time, as well as the abundance of disturbances to the mechanical properties of main arteries may be determined by different pathologic processes, for example, chronic renal disease, diabetes, rheumatoid arthritis and others. ${ }^{13-15}$ Some researchers also report increasing stiffness of main arteries in COPD. ${ }^{12,16}$

Specific changes in the position of some gene loci are also associated with the risk of appearance and development of COPD and asthma. ${ }^{17-19}$ Unlike monogenic diseases, the predisposition to COPD is determined by adaptive effect of several loci. Multiple gene alleles are not thought to be responsible for a pathological phenotype by itself, but their combination becomes pathogenic under the influence of inflammatory factors of the environment. Important genes for the development of COPD and asthma, regulate the plasma level of IgE, cytokines and chemokines, genes of microsomal epoxide hydrolase, glutathione-transferase, antichimotripsin-a1, complement GcG20 inclu- 
ding polymorphism of collagen-1 type 1a gene (COL1A1). 11,12,17,19-21 Polymerase chain reaction is the methodology used to confirm the promoted expression of the COL1A1 gene, related to the aberrant process of synthesis and degradation of extra-cellular type 1 collagen seen in COPD..$^{20,22}$ Studies have also described the relationship of polymorphisms of the COL1A1 gene and increased arterial stiffness in a group of healthy individuals. ${ }^{15,23}$ The same genetic polymorphism with close relationship and development of osteoporosis has been identified. Some polymorphisms and mutations of the COL1A1 gene are responsible for the development of connective-woven dysplasia and imperfect osteogenesis. ${ }^{24}$ In this regard, there is interest in the study of collagen type I gene polymorphism in patients with COPD and asthma, and the evaluation of its role in mitigating the establishment of arterial stiffness.

\section{Discussion}

Vascular disorders in COPD patients have been reported as endothelial dysfunction with disturbance of endothelium-dependent vasomotor reactions. These abnormalities may be connected with systemic inflammation, now recognized as a typical feature of COPD and cardiovascular comorbidity and part of the cardio respiratory continuum. ${ }^{14,16,17}$ A surplus of arterial stiffness is closely connected with disintegration of extra cellular matrix in arterial vessel walls, where an important component is collagen-1. Collagen-1 is an essential part of connective and bone tissue. ${ }^{12,15}$ Systemic inflammation in COPD disseminates not only in the lung but distributes to arterial vessels and promotes remodeling with growth in the intimae, increasing the amount of smooth muscle cells, depositing collagen and increasing the thickness and stiffness of arterial vessel walls. ${ }^{16,17}$

Progression of COPD is not only associated with increasing collagen deposition, but with disturbance of the structure and balance between different types of collagen in both the respiratory and vascular system. ${ }^{18}$ Thus, some COPD patients show disintegration of bronchial cartilage with replacement of bone tissue at the same time. One reason for this appears to be a result of the synthesis of collagen-1 by the connective tissue cells - the main structural part of bone..$^{18}$ This suggestion is partly supported by recent investigations showing that injection of TGF-b - one of the main vessel remodeling factors in rats, resulted in an increasing level of matrix RNA of collagen-1 and collagen- $3 .{ }^{25}$ Similarly, arterial vessel remodeling in asthma has been described - with typical features, included disturbance of angiogenesis with greater wall thickness, friability, and blood filling related with the bronchial walls. ${ }^{14}$ At the same time there is excess destruction of muscle and connective tissue, particularly filaments of collagen 1, 3 and 5 types in the lamina reticular is of basal membrane and sub-epithelial mucosa laminal of bronchi and vessels in severe asthma. ${ }^{26-28}$ Unlike COPD, there is no significant increasing the stiffness of the aorta in asthmatics, including severe cases of the disease. ${ }^{19}$

As previously reported, excess arterial stiffness is commonly observed in COPD, irrespective of whether or not the COPD is characterized as stable or unstable disease. The abundance of arterial stiffness may relate to irreversible processes in the aortic walls, however. ${ }^{6,14} \mathrm{At}$ the same time there is no direct correlation between increasing arterial stiffness and decline of expiratory volume flow. In the cited studies, increasing arterial stiffness in GOLD I and GOLD II patients has been estimated, but in GOLD III it has not been well characterized. These results may relate to remodeling of the left and right heart chambers and vessels with development of diastolic dysfunction and more fre- quent presence of hypoxemia in GOLD III patients. ${ }^{13,29}$ Permanent and excessive arterial stiffness in COPD patients can be considered as a feature of increasing of cardio-vascular risk and one of the reasons for reclassification of risk degree. ${ }^{7,13}$ At the same time, in asthma patients the degree of arterial stiffness has been reported to differ between remission rates and exacerbation rates of asthma. The aortic stiffness markedly increased during exacerbation and returned to normal during remission of asthma. It is theorized that increasing aortic stiffness during exacerbations of asthma promotes dysfunction of the elastic features of the aorta, serving as a pathophysiologic process. ${ }^{19}$

Our research showed an increase in the incidence of the functionally less restrictive allele of the S polymorphism of the COL1A1 gene in COPD patients, compared to the control group (healthy volunteers). At the same time, a decrease in the frequency of the dominant $\mathrm{S}$ allele of the same polymorphism was found, especially in patients with GOLD III and IV stage COPD classification. ${ }^{19,20,30}$ As a percentage of healthy persons, the distribution of the SS-Ss-ss genotypes was 92.1\% - 7.9\% - $0 \%$, and in COPD patients, respectively: $29.3 \%-40.3 \%$ $-30.4 \%$. There was no homozygosity of the $\mathrm{S}$ allele in patients with COPD IV by GOLD. Detection of the ss genotype of the COL1A1 gene in patients with severe COPD (GOLD IV) was extremely high. In the control group, there were no patients homozygous on the restrictive allele (ss) of the polymorphism Sp1 of the COL1A1 gene.

The distribution of gene COL1A1 alleles in patients with asthma differs from that in COPD. The number of restrictive $\mathrm{s}$ alleles was increased only in patients with severe asthma. Unlike in COPD, in severe asthma decreasing the amount of normal alleles $\mathrm{S}$ was due to increasing the number of heterozygotes Ss, which reached $81,9 \%{ }^{20}$ Pathologic genotype was registered only in $12,5 \%$ of patients with severe asthma, less often than in COPD $(p<0,01)$. At the same time the rate of occurrence of $\mathrm{S}$ and $\mathrm{s}$ alleles in the group with moderate asthma was the same as that in controls. So we can assume that the occurrence of insufficient $\mathrm{s}$ alleles of the COL1A1 gene increased only in patients with severe asthma, mostly because of heterozygous genotype Ss.

The desire to study the Sp1 polymorphism of COL1A gene in patients with COPD and the associated mechanical disturbances of the aorta is not a casual interest. The COL1A1 gene codes the amino acid structure of collagen type1, which is an important part of the extracellular matrix of the main artery walls. ${ }^{15,23}$ Polymorphisms in the regulatory area of the COL1A1 gene increase the level of its transcription and change the balance of $\alpha 1$ и $\alpha 2$ chains of protein, followed by a destructive process in the collagen of the arterial vessel wall. An imbalance of the protease-antiprotease system, which is seen in COPD also supports this pathologic process, much like a deficit of $\alpha 1$-antitrypsin. ${ }^{16,17}$ The cumulative result of these path ways are disturbances in the mechanical features of the artery walls in patients with COPD, which correlate with the decline of expiratory flow and clinical course of the disease. ${ }^{13,14}$

Analysis of relations between polymorphism Sp1 of COL1A1 gene and markers of arterial stiffness were made. The distribution of genotypes SS - Ss - ss in healthy people were: $92,1 \%-7,9 \%-0 \%$, and in patients with COPD: 29, 3\% - 40, 3\% - 30, 4\% accordingly. ${ }^{20}$ Parameters of aortic stiffness in healthy people with genotype SS and Ss were not so different, but in patients with COPD the differences between average parameters of APWV with genotypes SS, Ss, ss were significant $(p<0,01)$. Allele $s$ in homozygous and heterozygous position was associated with high parameters of aortic stiffness. These 
data shows that in persons with insufficient allele s the balance between collagen synthesis in the vessel wall and degradation is disordered. The differences between the levels of APWV in persons with genotypes Ss and ss were not revealed. Allele S in homozygous position was associated with normal artery stiffness. There is an opinion that allele s of Sp1 polymorphism of COL1A1 gene is connected with the markers of artery stiffness; we can conclude that this allele has an important part in main arteries stiffness disturbances in persons with COPD. At the same time the relations between Sp1 polymorphism of COL1A1 gene and the markers of arterial stiffness in persons with asthma were analyzed. The significant connection between APWV and allele s or ss as variant of COL1A1 gene was not revealed. There were not differences in aortic mechanical characteristics (stiffness) in remission phase of the disease in subgroups of patients with COL1A1 genotypes.

So, the differences in morphologic and functional conditions of the vessels in patients with COPD and asthma, may be connected with the features of genotype, particularly with especial distribution of Sp1 polymorphism COL1A1 gene. It's clear that this gene do not have a main meaning in determination of increased arterial stiffness in COPD, the other polymorphisms influences are not excluded. ${ }^{6,17,19}$ Never the less, one of the factors of forming the specific stable remodeling of pulmonary and other basic arteries in the case of COPD ${ }^{16,17}$ are the specific features of COL1A1 gene genotype described by us. The vessels remodeling, specific for asthma, ${ }^{11}$ in which there is no unreturnable increasing of arteries stiffness, also corresponds to our results. Our studies demonstrate the presence of association of insufficient alleles of COL1A1 gene with the markers of arterial stiffness in COPD. The analysis of some genetic factors influence on elastics of basic arteries in different pathologic conditions, shows its importance in understanding of pathologic mechanisms of increasing arterial stiffness. The testing of genes-candidates to be specific for COPD and increasing of aorta's stiffness opens real opportunities for earlier prophylactic of arterial stiffness changes. We conclude that authentic difference was established in genotype distribution between COPD patients and healthy persons. Functionally defective allele S (Sp1 polymorphism of COL1A1) was registered much oftener. Presence of it's homozygote variant $\mathrm{S}$ was closely associated with severe degree of COPD patients. COPD patients allele s (polymorphism Sp1 of gene COL1A1), associated with collagen metabolism disorders, is closely connected with surplus aortal stiffness. This underlines prognostic significance of allele SS in early diagnosis of large artery disorders.

Based on an analysis of literature data and own research, the authors provide a consensus view on the pathogenesis of persistent increases in arterial rigidity in COPD (Figure 1). The dysregulation of the collagen-elastin balance caused by various factors (classical inflammatory, oxidase and disproteinase pathways are considered) leads to an increase in vascular rigidity. ${ }^{13,15,19}$ This can explain the progressive deterioration of the mechanical properties of the central arteries in COPD patients, since this disease is precisely the universal aggression factors associated with persistent systemic inflammation, oxidative stress, imbalance in the proteinase inhibitors system. This is confirmed by the data of our study showing the heterogeneity of the structure of the walls of large arteries in experimental COPD with the zones of formation of connective tissue, among which collagen predominates. At the same time, in patients with asthma, the structure of the walls of large arteries remains more stable in comparison with COPD, although it is characterized by peculiar signs of remodeling (swelling of the endothelium, hypertrophy of collagen fibers and myocytes). ${ }^{31}$

Also, the authors provide an agreed opinion on the clinical effects of abnormal (increased) arterial stiffness (Figure 2). With COPD, heart and vascular remodeling is developing, one of the main characteristics of which is to increase their rigidity. The increase in stiffness of both the aorta and the myocardium is now recognized as a universal response to the effect of a variety of damaging factors. ${ }^{6,7,10,15}$ These changes entail a number of unfavorable pathophysiological and clinical consequences. Excessive stiffness of the aorta is a reliable independent predictor of general and cardiovascular mortality. ${ }^{7,8}$ Loss of vascular elasticity is one of the main factors in the progression of cardiovascular risk. Increasing rigidity of the central arteries leads to decreasing ability of the vascular system to respond adequately to the changing conditions of hemocirculation, the increase in postload on the myocardium and the disturbance of coronary blood supply. ${ }^{6,13,15}$ Hypertrophy and increased stiffness of the LV increase the need for myocardium in oxygen and increase the risk of myocardial infarction, heart failure, sudden death. ${ }^{32,33}$ Thus, excessive stiffness of the aorta and myocardium of the LV cause a significant increasing of the total cardiovascular risk in COPD patients. ${ }^{2,16,19}$ In addition, according to recent data, excessive arterial stiffness contributes to the pathogenesis of cerebral discirculation. ${ }^{31,34}$ Known the nature of the formation and high clinical significance, myocardium and central arteries rigidity increasing can be regarded as a systemic manifestation of COPD.

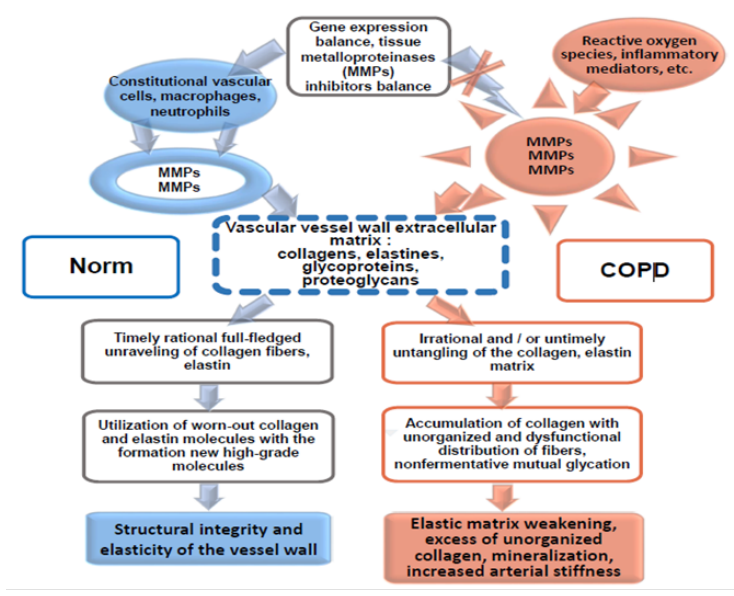

Figure I Pathogenesis of persistent increase of arterial stiffness in COPD. 


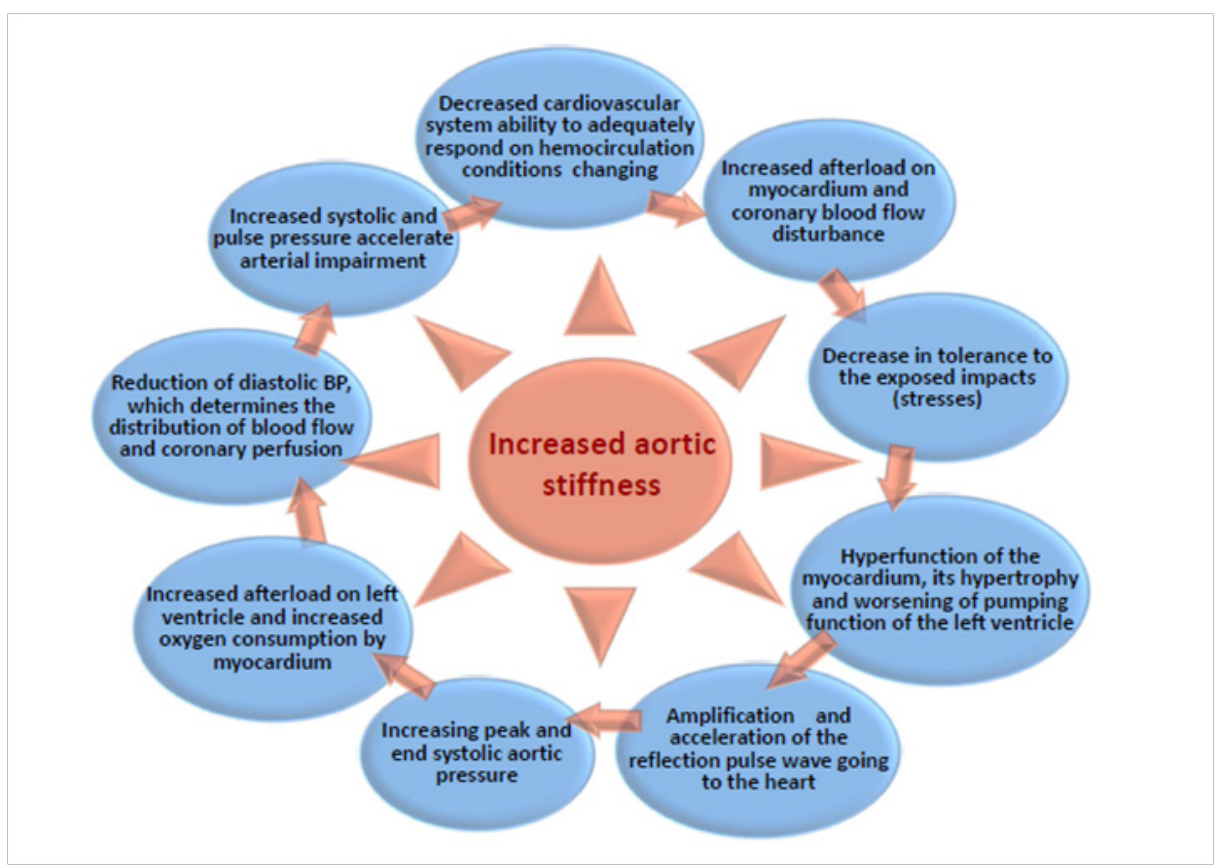

Figure 2 Clinical effects of increasing arterial stiffness: a vicious cycle.

\section{Conclusion}

The disturbance of the aortic mechanical features is a typical part of COPD pathophysiology. According to literature data and our own results we conclude that authentic modification observed in genotype distribution between COPD patients and healthy persons. Different genotypes are involved in aortic mechanical properties and other vascular disturbances in COPD. In particular functionally defective allele s (polymorphism Sp1 of gene COL1A1), associated with collagen metabolism disorders, is closely connected with surplus aortal stiffness in COPD patients. Genetic features and other disorders cause abnormalities in the mechanical properties of the aorta in COPD, as ordered in the scheme. In the following turn, increased aortic stiffness is an important component of increasing total cardiovascular risk, cardiovascular events, coronary disorders and cerebral distinction in COPD patients. ${ }^{35,36}$

\section{Acknowledgments}

Study was supported by Pacific State Medical University grant AAAA-A16-116070610052-3.

\section{Conflict of interest}

Author has declared there is no conflict of interest.

\section{References}

1. Bhatt SP, Cole AG, Wells JM, et al. Determinants of arterial stiffness in COPD. BMC Pulm Med. 2014;14:1.

2. Sin DD, Man SF. Chronic obstructive pulmonary disease as a risk factor for cardiovascular morbidity and mortality. Proc Am Thorac Soc. 2005;2(1):811.

3. Sabit R, Bolton CE, Edwards PH, et al. Arterial stiffness and osteoporosis in chronic obstructive pulmonary disease. Am J Respir Crit Care Med. 2007;175(12):1259-1265.

4. Oda M, Omori H, Onoue A, et al. Association between airflow limitation severity and arterial stiffness as determined by the brachial-ankle pulse wave velocity: a cross-sectional study. Intern Med. 2015;54(20):2569-2575.

5. Tabara Y, Muro S, Takahashi $\mathrm{Y}$, et al. Airflow limitation in smokers is associated with arterial stiffness: the Nagahama Study. Atherosclerosis. 2014;232(1):59-64.

6. Van Bortel LM, Laurent S, Boutouyrie P, et al. Expert consensus document on the measurement of aortic stiffness in daily practice using carotid-femoral pulse wave velocity. J Hypertens. 2012;30(3):445-448.

7. Vasyuk UA, Ivanova SV, Shkolynik EL, et al. Consensus of Russian experts coordinated opinion on the arterial stiffness assessment in clinical practice. Cardiovascular therapy and prevention. 2016;15(2):4-19.

8. Laurent S, Cockcroft J, Van Bortel L, et al. Expert consensus document on arterial stiffness: methodological issues and clinical applications. Eur Heart J. 2006;27(21):2588-2605.

9. Sakovskaia A, Nevzorova V, Brodskaya T, et al. Condition aortic stiffness and content of adipokines in the serum of patients with essential hypertension in young and middle-aged. Journal of Hypertension. 2015;33(S1):182.

10. Barton M, Husmann M, Meyer MR. Accelerated Vascular Aging as a Paradigm for Hypertensive Vascular Disease: Prevention and Therapy. Can J Cardiol. 2016;32(5):680-686.

11. Dolinchuk LV, Basanets AV, Andrushchenko TA. Genetic aspects of chronic obstructive pulmonary disease development. Ukraïnskiizhurnal z problem meditsinipratsi. 2013;1(34):44-56.

12. Yang L, Li X, Tong X, et al. Association between glutathione S-transferase P1 Ile (105) Val gene polymorphism and chronic obstructive pulmonary disease: A meta-analysis based on seventeen case-control studies. Meta Gene. 2015;6:59-64.

13. Nevzorova VA, Brodskaya TA, Golotina OV, et al. Cardiovascular dysfunction in chronic obstructive pulmonary disease and ischemic heart disease (functional metabolic parallels). Vladivostok: Dalnauka; 2015. p. 160 .

Citation: Brodskaya T, Nevzorova V, Zakharchuk N, et al.Aortic stiffness and polymorphisms of collagen-I type la gene in COPD patients.J Lung Pulm Respir Res. 2018;5(3):8I-85. DOI: I0.15406/jlprr.2018.05.00167 
14. Geltser BI, Brodskaia TA, Nevzorova VA. Arterial rigidity and chronic obstructive pulmonary disease: pathophysiological correlations and clinical implications. Ter Arkh. 2008;80(11):89-94.

15. Zieman SJ, Melenovsky V, Kass DA. Mechanisms, Pathophysiology, and Therapy of Arterial Stiffness. Arterioscler Thromb Vasc Biol. 2005;25(5):932-943.

16. Nevzorova VA, Brodskaya TA, Geltser BI. Arterial rigidity as a factor in increasing the level of cardiovascular risk in patients with COPD (pathophysiological mechanisms and clinical significance) In: Shumatov VB, Nevzorova VA, editors. Clinical pathophysiology of systemic manifestations of COPD. Vladivostok: Medicine DV; 2012. p. 29-91.

17. Global Initiative for Chronic Obstructive Lung Disease. Global Strategy for the Diagnosis, Management, and Prevention of Chronic Obstructive Pulmonary Disease. 2016

18. Mikhaleva LM, Cherniaev AL, Bykanova AV, et al. Morphofunctiona characteristics of pulmonary and bronchial arteries in bronchial asthma, chronic obstructive lung disease, idiopathic fibrosingalveolitis. Arkh Patol. 2008;70(1):35-37.

19. Brodskaya TA, Geltser BI, Nevzorova VA. Arterial stiffness and respiratory deseases (pathophysiological mechanisms and clinical significance). Vladivostok: Dalnauka; 2008. p. 248.

20. Geltser BI, Brodskaya TA, Nevzorova VA, et al. Analysis of the relationship between the genotype COL1A1 and markers of arterial stiffness in patients with COPD and bronchial asthma. Problems of tuberculosis and respiratory diseases. 2008;7:43-46.

21. Lakhdar R, Denden S, Knani J. et al. Combined analysis of EPHX1, GSTP1, GSTM1 and GSTT1 gene polymorphisms in relation to chronic obstructive pulmonary disease risk and lung function impairment. Dis Markers. 2011;30(5):253-263.

22. Ning W, Li CJ, Kaminski N, et al. Comprehensive gene expression profiles reveal pathways related to the pathogenesis of chronic obstructive pulmonary disease. PNAS. 2004;101(41):14895-14900.

23. Brull DJ, Murray LJ, Boreham CA, et al. Effect of a COL1A1 Sp1 Binding Site Polymorphism on Arterial Pulse Wave Velocity. An Index of Compliance. Hypertension. 2001;38(3):444-448.

24. Geltser BI, Kochetkova EA, Grigor'eva OIu, et al. Relations between VDR3 and COL1A1 genes and markers of bone tissue metabolism in patients with chronic obstructive pulmonary disease. Ter Arkh. 2006;78(3):17-20.

25. Kenyon NJ, Ward RW, McGrew G, et al. TGF-ß1 causes airway fibrosis and increased collagen I and III mRNA in mice. Thorax. 2003;58(9):772-777.

26. The ENFUMOSA Study Group. The ENFUMOSA cross-sectional Europian multicentre study of the clinical phenotype of chronic severe asthma. Eur Respir J. 2003;22(7):470-477.

27. Jeffery PK. Remodeling and Inflammation of Bronchi in Asthma and Chronic Obstructive Pulmonary Disease. Proceedings of the ATS 2004;1(3):176-183.

28. Ward C, Pais M, Bish R, et al. Airway inflammation, basement membrane thickening and bronchial hyperresponsiveness in asthma. Thorax. 2002;57(4):309-316.

29. Nevzorova VA, Golotina OV, Kochetkova EA, et al. Intracardiac hemodynamics in patients with isolated chronic obstructive pulmonary disease and coronary heart diseas. Klin Med (Mosk). 2010;88(1):37-41.

30. Nevzorova V, Vakhrusheva S, Tilik T, et al. Polymorphism of GSTP and EPHX1 genes in smokers and patients with $\mathrm{i}$ and ii stages of chronic obstructive pulmonary disease. European Respiratory Journal. 2012;40(S56):776

31. Geltser BI, Brodskaya TA, Kotelnikov VN, et al. Endothelial dysfunction of cerebral and major arteries during chronic obstructive disease. Experimental Biology and Medicine. 2007;144(6):768-771.

32. Stanton T, Jenkins C, Haluska BA, Marwick TH. (2014) Association of outcome with left ventricular parameters measured by two-dimensional and three-dimensional echocardiography in patients at high cardiovascular risk. J Am Soc Echocardiogr. 2014;27(1):65-73.

33. Pérez-Riera AR, Barbosa-Barros R, Shenasa M. Electrocardiographic Markers of Sudden Cardiac Death (Including Left Ventricular Hypertrophy). Card Electrophysiol Clin. 2017;9(4):605-629.

34. Nevzorova VA, Zakharchuk NV, Agafonova IG, et al. Endotheliumdependent and endothelium-independent cerebral vessels reactions in a chronic smoking model. International Journal of Biomedicine. 2011;2:103-107.

35. Cho MH, McDonald ML, Zhou X, et al. Risk loci for chronic obstructive pulmonary disease: a genome-wide association study and meta-analysis. The Lancet Respiratory Medicine. 2014;2(3):214-225.

36. Castaldi PJ, Cho $\mathrm{MH}$, Cohn $\mathrm{M}$, et al. The COPD genetic association compendium: a comprehensive online database of COPD genetic associations. Hum Mol Genet. 2010;19(3):526-534. 